\title{
Further test of internal-conversion theory with a measurement in ${ }^{197} \mathbf{P t}$
}

\author{
N. Nica, ${ }^{*}$ J. C. Hardy ${ }^{\dagger}$ V. E. Iacob, ${ }^{*}$ J. Goodwin, C. Balonek,${ }^{\ddagger}$ M. Hernberg,,${ }^{\S}$ and J. Nolan ${ }^{\|}$ \\ Cyclotron Institute, Texas A\&M University, College Station, Texas 77843, USA
}

\author{
M. B. Trzhaskovskaya \\ Petersburg Nuclear Physics Institute, Gatchina RU-188300, Russia \\ (Received 11 November 2009; published 16 December 2009)
}

\begin{abstract}
We have measured the $K$-shell internal conversion coefficient, $\alpha_{K}$, for the $346.5-\mathrm{keV} M 4$ transition in ${ }^{197} \mathrm{Pt}$ to be 4.23(7). This result differs from a previous value, which disagreed significantly from theory. Our new value agrees well with Dirac-Fock calculations and removes the earlier discrepancy as a source of concern.
\end{abstract}

DOI: 10.1103/PhysRevC.80.064314

PACS number(s): 23.20.Nx, 27.80.+w

\section{INTRODUCTION}

Internal conversion plays an important role in most nuclear decay schemes yet, until recently, the accuracy of calculated internal conversion coefficients (ICCs) was, at best, ill defined. Not only were there very few precise measurements of ICCs in existence but, for many years, there appeared to be a systematic difference between experiment and theory. In 1973 Raman et al. [1] compared "precisely measured" ICCs for 15 E3 and M4 transitions with the tabulated Hager and Seltzer calculations [2] and concluded that the theoretical values were systematically higher by $2-3 \%$. However, even this select group of transitions included only five with measured ICCs that were known with a precision of $2 \%$ or better, so the apparent discrepancy was hardly definitive. Even so, this is where the matter remained for 30 years.

By 2002, Raman et al. [3] had 100 experimental ICCs to compare with tabulated values, but even at that recent date only 20 of the measured ICCs had a precision of $2 \%$ or better. Their results still indicated that all previous tables of ICCs exhibited a $3 \%$ systematic bias but the authors found much better agreement (within $\sim 1 \%$ ) for a new table by Band et al. [4], which was calculated in the framework of the Dirac-Fock method, with the exchange between electrons treated exactly. Yet, even though the average agreement was now much better, some of the individual ICCs disagreed significantly with the calculated values and, even more troubling, the data appeared to show a surprising preference for one particular model in which the final-state electron wave function was computed in a field that did not include the atomic subshell vacancy caused by the conversion process.

\footnotetext{
*On leave from the National Institute for Physics and Nuclear Engineering "Horia Hulubei," Bucharest, Romania.

†hardy@comp.tamu.edu

${ }^{\ddagger}$ REU summer student from State University of New York at Buffalo, Buffalo, NY, USA.

${ }^{\S}$ REU summer student from State University of University of Iowa, Iowa City, IA, USA.

"REU summer student from Centenary College of Louisiana, Shreveport, LA, USA.
}

The question of whether or not to include the atomic vacancy was settled in 2004 by our precise measurement $( \pm 0.8 \%)$ of the $K$-shell conversion coefficient, $\alpha_{K}$, for the 80.2-keV M4 transition in ${ }^{193} \operatorname{Ir}[5,6]$ and was subsequently confirmed by our measurements of $\alpha_{K}$ for the $127.5-\mathrm{keV}$ E3 transition in ${ }^{134} \mathrm{Cs}$ and the $661.7-\mathrm{keV} M 4$ transition in ${ }^{137} \mathrm{Ba}[7,8]$. The impact of these results is illustrated in Fig. 1, where we plot differences between 21 experimental ICCs and two versions of the Dirac-Fock theory (see $[3,5]$ ), one that ignores the atomic vacancy and the other that includes it via the "frozen orbital" approximation. In addition to our three results, the figure includes the 20 cases with better than $2 \%$ precision, which were listed by Raman et al. [3] in 2002. Note that two of the Raman results have been supplanted by our new measurements. Clearly our results leave little doubt that the atomic vacancy must be incorporated in the ICC calculation.

Nevertheless, there remain two cases-the 39.8-keV E3 transition in ${ }^{103} \mathrm{Rh}$ and the $346.5-\mathrm{keV} M 4$ transition in ${ }^{197} \mathrm{Pt}-$ that differ by more than two standard deviations from both types of calculation. If taken at face value, these two discrepant cases could indicate that there is some further aspect of the theory that is not quite complete. In this paper we address one of these apparently discrepant cases, the $M 4$ transition in ${ }^{197} \mathrm{Pt}$. We report a measurement of its $\alpha_{K}$ value to a precision of $1.7 \%$.

\section{MEASUREMENT OVERVIEW}

We have described our measurement techniques in detail in previous publications [5,7] so only a summary will be given here. If a decay scheme is dominated by a single transition that can convert in the atomic $K$ shell, and a spectrum of $K$ x rays and $\gamma$ rays is recorded for its decay, then the $K$-shell internal conversion coefficient for that transition is given by

$$
\alpha_{K} \omega_{K}=\frac{N_{K}}{N_{\gamma}} \cdot \frac{\epsilon_{\gamma}}{\epsilon_{K}},
$$

where $\omega_{K}$ is the fluorescence yield; $N_{K}$ and $N_{\gamma}$ are the total numbers of observed $K \mathrm{x}$ rays and $\gamma$ rays, respectively; and $\epsilon_{K}$ and $\epsilon_{\gamma}$ are the corresponding detector efficiencies. Fluorescence yields have been well measured for tantalum $(Z=73)$ and mercury $(Z=80)$ [9], elements that bracket 


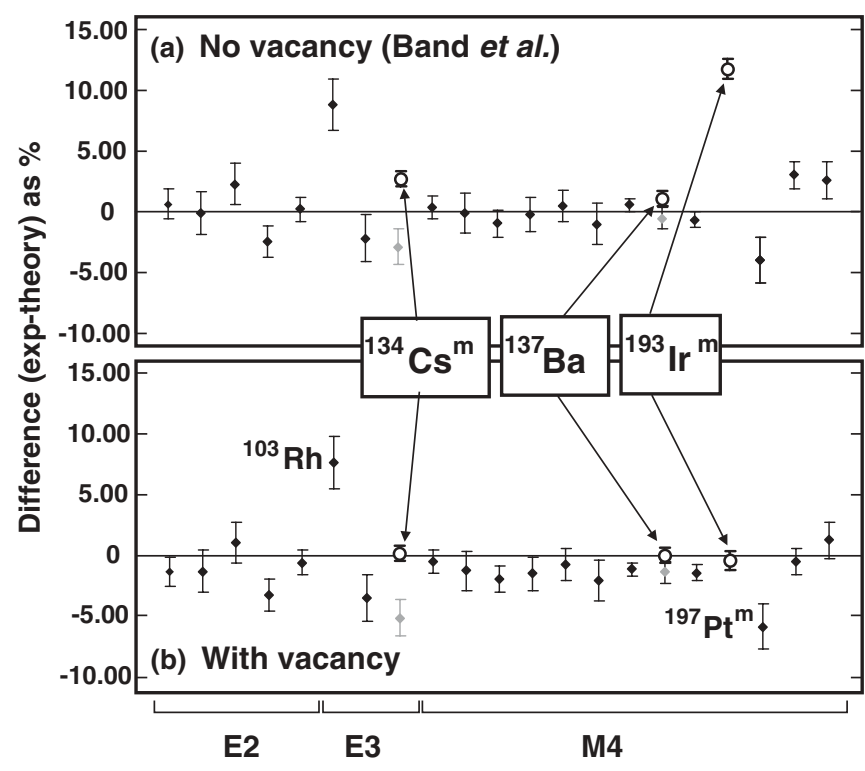

FIG. 1. Percentage differences between the measured and calculated ICCs for two Dirac-Fock calculations: one (a) is without the atomic vacancy and the other (b) is with it included in the "frozen orbital" approximation. The points shown as solid diamonds in both plots correspond to the 20 cases listed by Raman et al. [3] that have better than $2 \%$ precision; as indicated at the bottom, five are for $E 2$ tranditions, three for $E 3$, and the remainder are for $M 4$ transitions. The points shown as open circles correspond to our three more-recently measured $\alpha_{K}$ values. For the cases of ${ }^{134} \mathrm{Cs}^{m}$ and ${ }^{137} \mathrm{Ba}[7,8]$ the earlier Raman values are shown in grey; for ${ }^{193} \operatorname{Ir}^{m}[5,6]$ there was no earlier value with sub-2\% precision.

platinum $(Z=78)$. They have also been evaluated as a function of $Z$ [10] for all elements with $10 \leqslant Z \leqslant 100$, and $\omega_{K}$ values have been recommended, which have an uncertainty of about $\pm 0.4 \%$ at $Z=78$. Thus, in the mass region of interest here, Eq. (1) allows $\alpha_{K}$ to be extracted directly from measured peak areas with subpercent precision provided that the detector efficiencies are sufficiently well known.

In our experiments, we detect both the $\gamma$ ray and the $K$ $\mathrm{x}$ rays in the same HPGe detector, a detector whose efficiency has been meticulously calibrated [11-13] to subpercent precision between 50 and $3500 \mathrm{keV}$. In our present study of the $M 4$ transition in ${ }^{197} \mathrm{Pt}$, the $\gamma$ ray of interest is at $346.5 \mathrm{keV}$ and the platinum $K$ x rays lie between 64 and $78 \mathrm{keV}$. All are comfortably within the well-calibrated range, so the efficiency ratio, $\epsilon_{\gamma} / \epsilon_{K}$, in Eq. (1) can also be quoted to subpercent precision.

The decay scheme of the $95.41-\mathrm{min}$ isomer in ${ }^{197} \mathrm{Pt}$ is shown in Fig. 2. Its predominant decay path is via the cascaded transitions with energies of $346.5 \mathrm{keV}$ and $53.1 \mathrm{keV}$, the former being the $M 4$ transition of interest here. Both transitions convert, but the $53.1-\mathrm{keV}$ transition can only convert in the $L$ and higher shells. Thus the $K$-x-ray peak from platinum observed in a decay spectrum of ${ }^{197} \mathrm{Pt}^{m}$ can only be due to the conversion of the $346.5-\mathrm{keV}$ transition. This satisfies the "single transition" requirement for the validity of Eq. (1).

Unfortunately that is not the full story. As shown in Fig. 2, the transition cascade from the isomer populates the ${ }^{197} \mathrm{Pt}$

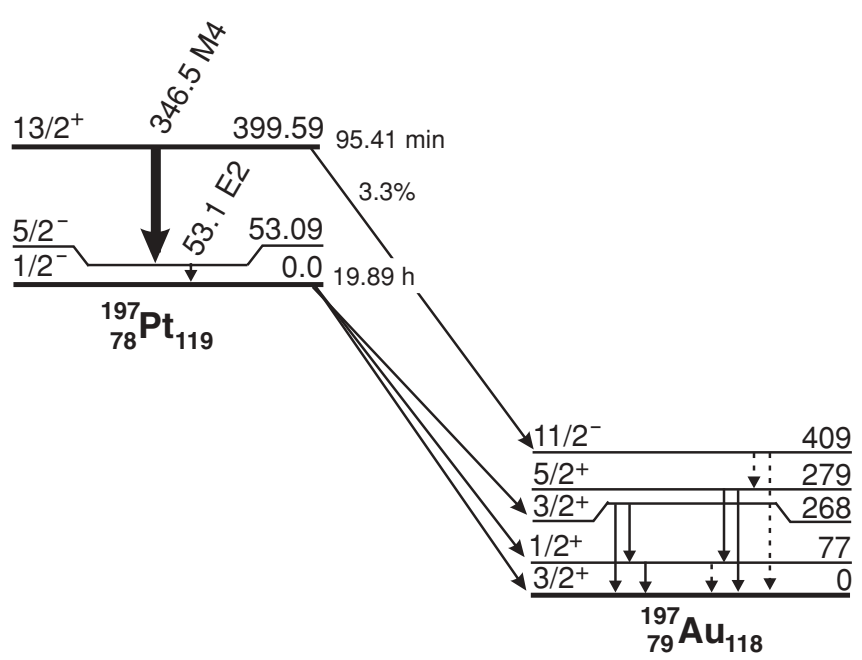

FIG. 2. Abbreviated decay scheme for the decay of ${ }^{197} \mathrm{Pt}^{m}$, showing both its $\gamma$-ray/internal-conversion transition and its $\beta$ decay. Also shown is the $\beta$ decay of the ${ }^{197} \mathrm{Pt}$ ground state, which was also produced in this experiment. The $\gamma$ transitions in ${ }^{197} \mathrm{Au}$ are grouped according to which $\beta$-decaying state in ${ }^{197} \mathrm{Pt}$ feeds them. The group of three on the left corresponds to the ${ }^{197} \mathrm{Pt}$ ground-state decay; the group of five on the right corresponds to the isomeric-state decay. The dashed arrows represent very weak $\gamma$ rays. All the information is taken from Ref. [14].

ground state, which itself is radioactive and decays with a 19.89-hr half-life by $\beta$ decay to two excited states and the ground state in ${ }^{197} \mathrm{Au}$. Furthermore, the 95.41-min isomer also has a $3.3 \% \beta$-decay branch, which feeds a third excited state in ${ }^{197} \mathrm{Au}$. All three excited states populated in ${ }^{197} \mathrm{Au}$ decay by transitions that convert, although one of them-the $77-\mathrm{keV}$ transition from the first excited state-is too low in energy to convert in the $K$ shell. The net result, though, is that gold $K \mathrm{x}$ rays are present in every decay spectrum, overlapping to some degree the $K$ x rays from platinum and complicating their analysis. Worse still, the $77-\mathrm{keV} \gamma$ ray itself lies directly on top of the $K_{\beta}$ x rays from platinum, making it impossible to separate one from another using the energy spectrum alone.

These complications made it essential for us to obtain sequential spectra in order to use the different half-lives of the ${ }^{197} \mathrm{Pt}$ ground state and the $399.6-\mathrm{keV}$ isomeric state to untangle the contributions from the two decays. Ultimately, it is this extra step that limits our precision somewhat compared with what we have achieved previously [5-8]. It is also likely that the contributions from transitions in ${ }^{197} \mathrm{Au}$ have misled earlier authors [15] and resulted in the apparently anomalous behavior of the $\alpha_{K}$ value adopted by Raman et al. [3] for the M4 transition from ${ }^{197} \mathrm{Pt}^{m}$.

\section{EXPERIMENT}

We used the same experimental method and setup as in our previous measurements [5-8]. Only those details not covered in previous publications will be described here. 


\section{A. Source preparation}

We produced ${ }^{197} \mathrm{Pt}^{m}$ by neutron activation of a platinum sample enriched to $97.43(2) \%$ in ${ }^{196} \mathrm{Pt}$. The source material, which was purchased as metal powder from Trace Sciences International Corporation, was chemically pure, but contained small quantities of other stable platinum isotopes, with ${ }^{195} \mathrm{Pt}$ (1.6\%), ${ }^{194} \mathrm{Pt}(0.65 \%)$, and ${ }^{198} \mathrm{Pt}(0.32 \%)$ predominating.

Although the material came from the supplier as grains of metal, the grain size was large enough to cause significant self-attenuation of $\mathrm{x}$ rays relative to $\gamma$ rays, a serious concern for our measurement. Consequently we chemically processed the material in order to reduce the grain size. We placed a few drops of Aqua Regia-one part concentrated nitric acid combined with three parts concentrated hydrochloric acidon a clean microscope slide, and added a few grains of the enriched platinum metal, which weighed a total of $1.5 \mathrm{mg}$. The resulting solution was placed on a hot plate and kept at a steady $100^{\circ} \mathrm{C}$. The solution turned blood-red and then black, as intermediate compounds were formed, leading finally to the re-emergence of elemental platinum.

The powder that remained after all the liquid had evaporated was very fine grained but nonuniform and concentrated around the margin of the original drop. We obtained a more uniform layer, approximately $1 \mathrm{~cm}$ in diameter, by rearranging the grains with a spatula. We then picked this material up on the adhesive layer of a piece of $12.5-\mu \mathrm{m}$-thick Mylar tape, and sealed it with a second similar tape that we applied facing the first. Using a microscope we estimated that the metal powder covered about $20 \%$ of the total area of the source. This information, combined with the weight of the dissolved platinum, allowed us to deduce that the thickness of the platinum layer was about $4.5 \mu \mathrm{m}$, a result that we visually confirmed with the microscope by comparing the layer thickness with a tungsten wire of known $12.5 \mu \mathrm{m}$ diameter.

This sample assembly was placed for $1 \mathrm{~h}$ in a thermal neutron flux of $\sim 7 \times 10^{12} \mathrm{n} / \mathrm{cm}^{2} \mathrm{~s}$ at the TRIGA reactor in the Texas A\&M Nuclear Science Center. After removal from the reactor the sample was conveyed to our measurement location, where counting began $3 \mathrm{~h}$ after the end of activation.

\section{B. Radioactive decay measurements}

We acquired spectra with our precisely calibrated HPGe detector and with the same electronics used in its calibration [12]. Our analog-to-digital converter was an Ortec TRUMP $^{\mathrm{TM}}-8 \mathrm{k} / 2 \mathrm{k}$ card controlled by MAESTRO ${ }^{\mathrm{TM}}$ software. The TRUMP ${ }^{\mathrm{TM}}$ card uses the Gedcke-Hale method [16] to determine a live time that accounts for dead-time losses and random summing. In all, we obtained $278 \mathrm{k}$-channel spectra with a source-to-detector distance of $151 \mathrm{~mm}$, the distance at which our calibration is well established. Each spectrum covered the energy interval 10-2000 keV with a dispersion of about $0.25 \mathrm{keV} / \mathrm{channel}$. The first nine spectra were taken within six hours of the irradiation, a span that equals about four half-lives of ${ }^{197} \mathrm{Pt}^{m}$. The remaining spectra were obtained over an extended period of time- the last one after 13 days-so that we could confidently identify and correct for all relevant impurities. Acquisition times ranged from $14 \mathrm{~min}$ to $24 \mathrm{~h}$.

\section{ANALYSIS}

In our analysis of the data, we followed the same methodology as we did with previous source measurements [5-8]. We first extracted areas for all the $\mathrm{x}$ - and $\gamma$-ray peaks of interest. Then, we identified impurities, and made appropriate corrections to account for their effects on the peaks required for the determination of $\alpha_{K}$. Next we used a half-life analysis to remove the contribution from the $\beta$ decay of the ${ }^{197} \mathrm{Pt}$ ground state. Finally, we dealt with the various small corrections that had to be applied to the peak areas to account for coincidence summing, $\mathrm{x}$-ray attenuation and the non-Gaussian shape of the $\mathrm{x}$-ray peak.

\section{A. Peak areas}

The spectra in Fig. 3 show the energy regions of primary interest for this measurement, one encompassing the $K$ x rays and the other, the $346.5-\mathrm{keV} \gamma$ ray. As in our previous measurements [5-8], after subtraction of room background we determined all peak areas with GF2, the least-squares peak-fitting program in the RADWARE series [17]. In doing so, we used the same fitting procedures as were used in the original detector-efficiency calibration [11-13].

It can be seen that the $\gamma$-ray peak lies on a flat background, apparently well isolated from any other impurity peaks. However, the spectrum shown in the figure was taken shortly after the initial activation; later spectra indicated that there was a small contribution to the peak from a $344.3-\mathrm{keV} \gamma$ ray due to the decay of a 9.3-h ${ }^{152} \mathrm{Eu}^{m}$ impurity. In the next section we will describe how the size of the contribution was determined but, for now, it should simply be noted that subtraction of the

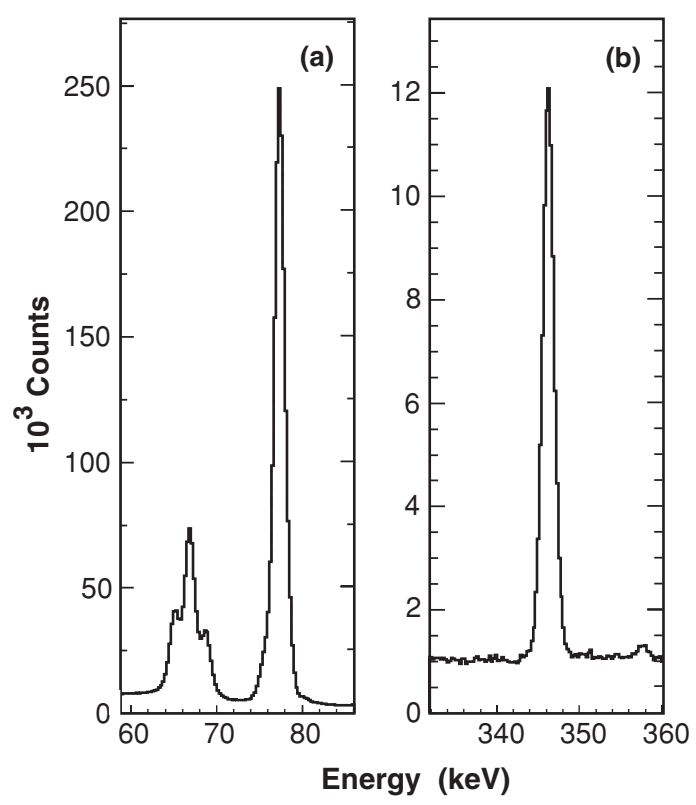

FIG. 3. Portions of the first spectrum collected after activation, covering the two energy regions of interest in the determination of the $\alpha_{K}$ ratio for the $346.5-\mathrm{kev}$ transition in ${ }^{197} \mathrm{Pt}$ : (a) the overlapping platinum and gold $K_{\alpha}$ x rays together with the $77.35-\mathrm{keV} \gamma$ ray from ${ }^{197} \mathrm{Au}$, which masks the $K_{\beta} \mathrm{x}$ rays; (b) the $346.5-\mathrm{keV} \gamma$ ray. 
flat background beneath the peak presents no difficulties for the peak analysis.

The x-ray region is not so simple. Clearly visible in the spectrum are the overlapping $K_{\alpha}$ peaks from both platinum and gold, the former being mostly from the $346.5-\mathrm{keV}$ transition of interest in ${ }^{197} \mathrm{Pt}$, the latter mostly from the $\beta$ decay of the ${ }^{197} \mathrm{Pt}$ ground state, which feeds levels in ${ }^{197} \mathrm{Au}$. The $77-\mathrm{keV}$ peak is one of the $\gamma$ rays from the latter decay and it completely masks all the $K_{\beta} \mathrm{x}$ rays. For obvious reasons, our analysis must depend strongly on the time-decay information from sequentially acquired spectra. To obtain peak areas in all spectra we included a very large energy region, approximately from 60 to $90 \mathrm{keV}$, and used a special modification [17] of the GF2 program that allows us to set a background by the normal fitting procedures and then to integrate the total number of counts above that background within set limits, extrapolating a Gaussian tail if needed to account for counts possibly outside those limits.

\section{B. Impurities}

In addition to the unavoidable contributions from the $\beta$ decays of ${ }^{197} \mathrm{Pt}$, we also observed a number of weak impurities in our sources, some coming from the sample itself and some from the Mylar substrate. As a typical example, a portion of one of our $27 \gamma$-ray spectra appears in Fig. 4 with contaminant peaks identified. This spectrum was acquired about 6 hours after the spectrum excerpted in Fig. 3, so the 346.5-keV $\gamma$ ray from the decay of the $95-\mathrm{min}{ }^{197} \mathrm{Pt}^{m}$ has already decayed by a factor of 16 compared to the earlier one. As is evident from the figure, even the weakest peaks were identified. We did the same for all recorded spectra and then carefully analyzed the results for contaminants. In all, we identified eight activities that make small contributions to our energy regions of interest. One, ${ }^{152} \mathrm{Eu}^{m}\left(t_{1 / 2}=9.31 \mathrm{~h}\right)$, produced a $\gamma$ ray at $344.3 \mathrm{keV}$, unresolved from the $346-\mathrm{keV}$ peak of interest from ${ }^{197} \mathrm{Pt}^{m}$; and the other seven contributed to the region of the platinum $K$ x rays: ${ }^{192} \mathrm{Ir}(73.8 \mathrm{~d}),{ }^{194} \mathrm{Ir}$ (19.3 h), ${ }^{195} \mathrm{Pt}^{m}$ (4.01 d), ${ }^{197} \mathrm{Hg}(64.1 \mathrm{~h}),{ }^{198} \mathrm{Au}(2.70 \mathrm{~d})$, ${ }^{199} \mathrm{Au}(3.14 \mathrm{~d})$, and ${ }^{199} \mathrm{Pt}$ (30.8 min). In addition, we observed ${ }^{24} \mathrm{Na},{ }^{38} \mathrm{Cl},{ }^{56} \mathrm{Mn},{ }^{122} \mathrm{Sb}$, and ${ }^{124} \mathrm{Sb}$ associated with the Mylar substrate, which do not interfere at all with our peaks of interest.

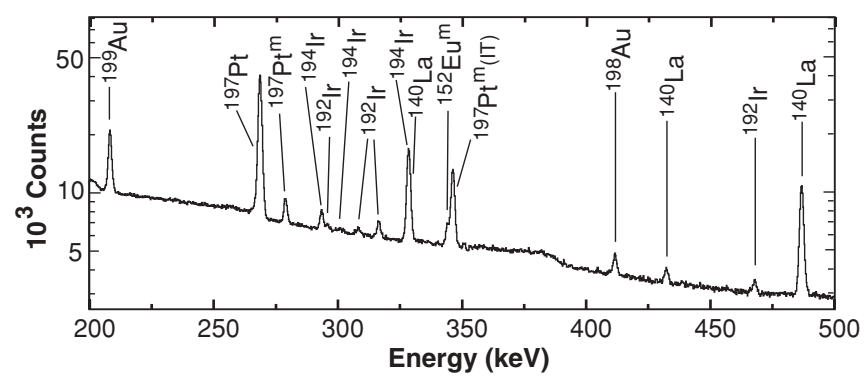

FIG. 4. Portion of the $\gamma$-ray energy spectrum measured $6 \mathrm{~h}$ after the spectrum shown in Fig. 3. Peaks are identified by their $\beta$-decay parent except for the $346.5-\mathrm{keV}$ peak, which is due to the isomeric transition from ${ }^{197} \mathrm{Pt}^{m}$.
TABLE I. The contributions of identified impurities to the energy regions of the $346.5-\mathrm{keV}$ peak and the platinum $K$ x-ray peaks. The activities, determined as decay rates at $t=0$, are expressed as percentages of the final, corrected rate at $t=0$ for the ${ }^{197} \mathrm{Pt}^{m}$ peaks to which they contribute.

\begin{tabular}{|c|c|c|}
\hline Source & Contaminant & $\begin{array}{l}\text { Relative activity } \\
\text { at } t=0(\%)\end{array}$ \\
\hline \multicolumn{3}{|c|}{ Contribution to $346.5-\mathrm{keV}$ peak: } \\
\hline${ }^{152} \mathrm{Eu}^{m}$ & 344.3-keV $\gamma$ ray & $0.65(7)$ \\
\hline \multicolumn{3}{|c|}{ Contribution to $K$ x-ray peaks: } \\
\hline${ }^{192} \operatorname{Ir}$ & Os + Pt $K$ x rays & $0.0187(14)$ \\
\hline${ }^{194} \mathrm{Ir}$ & Pt $K$ x rays & $0.0525(19)$ \\
\hline${ }^{195} \mathrm{Pt}^{m}$ & Pt $K$ x rays & $1.11(13)$ \\
\hline${ }^{198} \mathrm{Au}$ & $\mathrm{Hg} K$ x rays & $0.0045(4)$ \\
\hline${ }^{199} \mathrm{Au}$ & $\mathrm{Hg} K$ x rays & $1.56(16)$ \\
\hline${ }^{199} \mathrm{Pt}$ & $\mathrm{Au} K$ x rays & $0.33(3)$ \\
\hline${ }^{197} \mathrm{Hg}$ & $\mathrm{Au} K \mathrm{x}$ rays $+77.4-\mathrm{keV} \gamma$ ray & $0.29(14)$ \\
\hline
\end{tabular}

Only ${ }^{152} \mathrm{Eu}^{m}$ contributes to the $346.5-\mathrm{keV}$ peak. We saw evidence for it from two of its $\beta$-delayed $\gamma$ rays: one at $963.4 \mathrm{keV}\left(I_{\gamma}=11.7\right.$ per 100 decays $)$ and the other at $344.3 \mathrm{keV}$ (2.4 per 100 decays). We deduced its total activity by two independent methods: the first using the 963.4-keV $\gamma$ ray, which was cleanly observed; and the second using a half-life analysis of the unresolved $344.3-\mathrm{keV}$ and $346.5-\mathrm{keV}$ peaks. The two methods gave consistent results for the activity of ${ }^{152} \mathrm{Eu}^{m}$ at time $t=0$, which we defined to be the time at which counting began in the first spectrum. The result from the half-life analysis, which is the more precise, is given in Table I, where it is expressed as a percentage of the total corrected (i.e., final) activity of the $346.5-\mathrm{keV}$ peak at $t=0$. The time decay of the $346.5-\mathrm{keV}$ peak, after subtraction of the small ${ }^{152} \mathrm{Eu}^{m}$ contamination is shown (as filled triangles) in the bottom panel of Fig. 5.

In dealing with the impurities contributing to the platinum $K$ x-ray region, we obtained the contribution of each as a function of time, based on the observed intensities of $\gamma$ rays from that same impurity observed elsewhere in the spectrum. Those impurities that could be simply dealt with in this manner (and the energy of the $\gamma$-ray peaks we used) were ${ }^{192} \operatorname{Ir}(308.5$, $316.5 \mathrm{keV}),{ }^{194} \mathrm{Ir}(328.5 \mathrm{keV}),{ }^{195} \mathrm{Pt}^{m}(98.9,129.5,129.8 \mathrm{keV})$, ${ }^{198} \mathrm{Au}(411.8 \mathrm{keV}),{ }^{199} \mathrm{Au}(158.4 \mathrm{keV})$, and ${ }^{199} \mathrm{Pt}(543.0 \mathrm{keV})$. With the known half-life of each impurity, we fitted the areas of these peaks from all spectra in which they were observed and derived the activity of each at time $t=0$. Finally we obtained the activity, also at $t=0$, of the $K$ x rays for each impurity from their known relative $\mathrm{x}$-ray-to- $\gamma$-ray ratios [18]. The results are given in Table I, where they are expressed as percentages of the total corrected (i.e., final) activity of the summed platinum $\left(K_{\alpha}+K_{\beta}\right)$ x-ray peaks at $t=0$.

Only one of the contributing impurities, ${ }^{197} \mathrm{Hg}$, required more elaborate treatment since it decays by electron capture to ${ }^{197} \mathrm{Au}$ and produces the same $\gamma$ rays-with energies of 77.4 and $191.4 \mathrm{keV}$ - as does the ground-state $\beta$ decay of ${ }^{197} \mathrm{Pt}$. Without a unique signature of the presence of ${ }^{197} \mathrm{Hg}$, we were forced to use an analysis of the time-decay of the 191.4-keV 

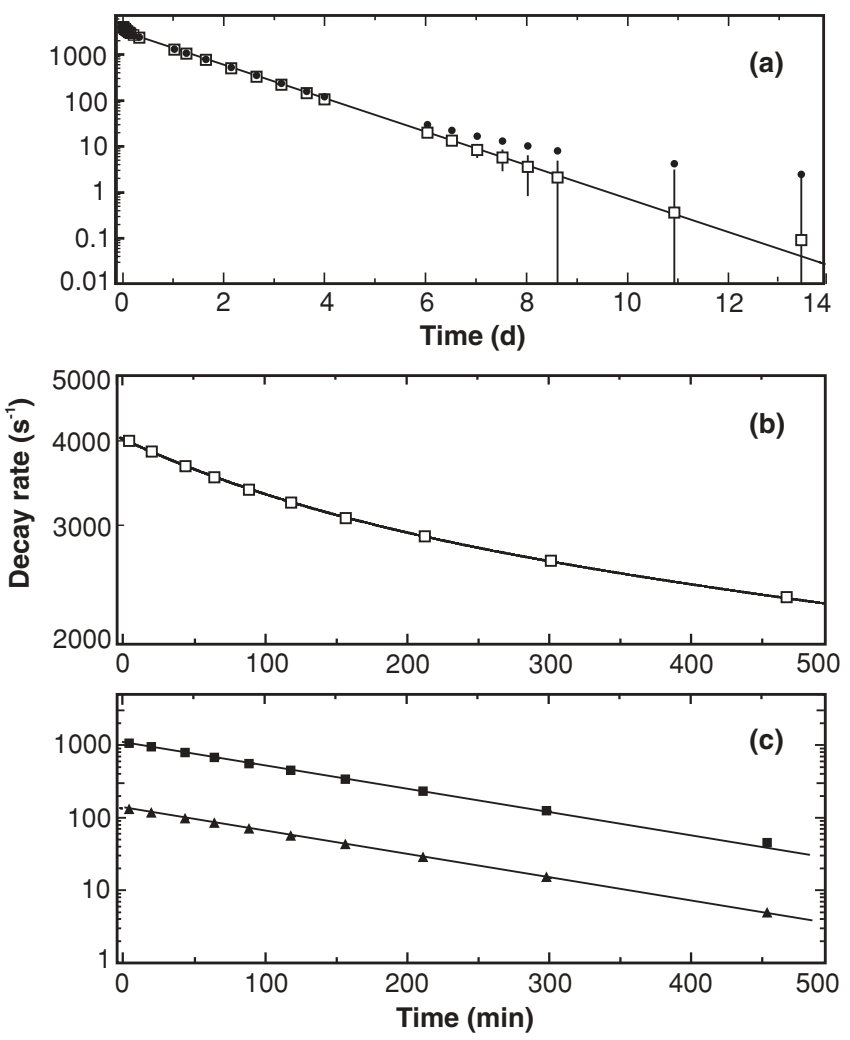

FIG. 5. All three panels present plots of decay rates as a function of time: (a) gives the rates obtained with the total, backgroundsubtracted counts in the energy region of the $K$ x rays (solid circles), together with the same results after further subtraction of the impurity contributions listed in Table I (open squares); (b) shows an expanded view of the first ten after-subtraction results from the top panel (again plotted as open squares). On the same expanded time scale, (c) presents (as solid squares) the results for the $K$ x rays from ${ }^{197} \mathrm{Pt}^{m}$ as obtained from a two-component fit to the composite decay in the middle and top panels. The corrected decay rates of the $346.5-\mathrm{keV}$ $\gamma$ ray from ${ }^{197} \mathrm{Pt}^{m}$ are also plotted (as solid triangles) in the bottom panel. Where no uncertainties are visible, they are smaller than the data points. All curves are computer fits with half-lives fixed to known values.

$\gamma$ ray to separate out the contribution of $64.1-\mathrm{h}{ }^{197} \mathrm{Hg}$ from that of the 95.4-min and 19.9-h states in ${ }^{197} \mathrm{Pt}$, which together lead to a growth and decay function. Fortunately, as can be seen in Table I, its impact on the $K$ x-ray region of the spectrum is very small. In fact, the total contribution from all impurities is only $3.4 \%$.

Once the impurities had been identified and their intensity in the region of the platinum $K$ x rays determined, we could subtract their contribution from the total area of the combined $K_{\alpha}$ and $K_{\beta}$ groups in each spectrum. The total areas, expressed as decay rates, are plotted against time in the top panel of Fig. 5, where they appear as filled circles. The decay rates, after the impurities have been subtracted, are shown in the same panel as open squares; and they appear again with the same symbols in the middle panel of the figure, in which only the first 500 minutes are shown to illustrate the region of particular interest for the ${ }^{197} \mathrm{Pt}^{m}$ decay.

\section{Competing transitions}

As remarked in Sec. II and illustrated in Fig. 2, our measurement is complicated by unavoidable competition from the $\beta$ decay of the ground state of ${ }^{197} \mathrm{Pt}$, which is produced directly by neutron activation and indirectly by the isomeric transition from ${ }^{197} \mathrm{Pt}^{m}$. This results in the strong production of gold $K$ x rays and of $77.4-\mathrm{keV} \gamma$ rays from de-excitation of the first excited state in ${ }^{197} \mathrm{Au}$. Both have the same behavior with time, exhibiting one decay component with the 19.9-h half-life from the decay of the ground-state ${ }^{197} \mathrm{Pt}$ nuclei present in the sample after activation, and a second growth-and-decay component caused by the subsequent production of the ground state by the decay of the isomer ${ }^{197} \mathrm{Pt}^{m}$.

We therefore fitted the after-subtraction results for the $K$ x-rays (open squares in the top and middle panels of Fig. 5) by including three components: the decay of ${ }^{197} \mathrm{Pt}^{m}$, the decay of the ${ }^{197} \mathrm{Pt}$ ground state, and the growth and decay of the latter fed by the former. Obviously only two half-lives were involved and we fixed these at the known values, 95.41(18) $\mathrm{min}$ for the isomer and 19.8915(19) h for the ground state [14]. The resulting fitted curve appears in the top two panels of the figure and displays excellent agreement with the data. For illustration purposes the bottom panel of the figure shows (as solid squares) the data after the fitted contribution from the ${ }^{197} \mathrm{Pt}$ ground-state $\beta$ decay has been subtracted. The line through the points is the corresponding fitted result with the known half-life of ${ }^{197} \mathrm{Pt}^{m}$. The resulting decay rate (evaluated at $t=0$ ) for the contribution of ${ }^{197} \mathrm{Pt}^{m}$ decay to the energy region of the $K \mathrm{x}$ rays is given in the top line of Table II.

TABLE II. Corrections to the ${ }^{197} \mathrm{Pt}^{m} K \mathrm{x}$ rays (upper section) and $346.5-\mathrm{keV} \gamma$ ray (middle section). The decay rates (at $t=0$ ) already corrected both for impurities (see Table I) and for the ${ }^{197} \mathrm{Pt}$ groundstate $\beta$ decay (see Sec. IV C) are given in the first row of each section, followed by the percentage adjustments for each listed effect. The corrected decay rates appear in the last row of each section. The lower section presents the final activity ratio together with the ingredients required to extract a value for $\alpha_{K}$. The symbols and notation are the same as in Eq. (1).

\begin{tabular}{|c|c|c|}
\hline Quantity & Value & Source \\
\hline \multicolumn{3}{|l|}{$\operatorname{Pt}\left(K_{\alpha}+K_{\beta}\right)$ x rays } \\
\hline Decay rate $\left(\mathrm{s}^{-1}\right)$ from ${ }^{197} \mathrm{Pt}^{m}$ & $1110(16)$ & Secs. IV B, IV C \\
\hline${ }^{197} \mathrm{Pt}^{m} \beta$ decay & $-1.9(1) \%$ & Sec. IV C \\
\hline Lorentzian correction & $+0.8(2) \%$ & Sec. IV F \\
\hline Corrected decay rate $N_{K}\left(\mathrm{~s}^{-1}\right)$ & 1098(16) & \\
\hline \multicolumn{3}{|l|}{${ }^{197} \mathrm{Pt}^{m} 346.5-\mathrm{keV} \gamma$ ray } \\
\hline Decay rate $\left(\mathrm{s}^{-1}\right)$ & $136.38(35)$ & Sec. IV B \\
\hline Coincident summing & $+2.1(4) \%$ & Sec. IV D \\
\hline Corrected decay rate $N_{\gamma}\left(\mathrm{s}^{-1}\right)$ & $139.24(64)$ & \\
\hline \multicolumn{3}{|l|}{ Evaluation of $\alpha_{K}$} \\
\hline$N_{K} / N_{\gamma}$ & $7.88(12)$ & This table \\
\hline Attenuation & $+1.4(5) \%$ & Sec. IV E \\
\hline$\epsilon_{\gamma} / \epsilon_{K}$ & $0.5077(10)$ & [12] \\
\hline$\omega_{K}$ & $0.959(4)$ & [10] \\
\hline$\alpha_{K}$ for $346.5-\mathrm{keV}$ transition & $4.23(7)$ & Eq. (1) \\
\hline
\end{tabular}


The bottom panel of Fig. 5 also shows the fit to the decay of the 346.5-keV line, in which the known half-life of ${ }^{197} \mathrm{Pt}^{m}$ was used. That fit, too, is excellent and the corresponding decay rate for the $\gamma$ ray, evaluated at $t=0$, is also listed in Table II.

The decay rate for the energy region of the $K$ x rays is predominantly due to the $M 4$ transition from ${ }^{197} \mathrm{Pt}^{m}$, which gives rise to the platinum $\mathrm{x}$ rays whose intensity we are seeking. However, it also includes a small contribution from the gold $\mathrm{x}$ rays and $77-\mathrm{keV} \gamma$ rays that follow the weak $\beta$-decay branch from the same isomeric state. We can determine that contribution from the ratio of the $279-\mathrm{keV} \gamma$ ray to the $346.5-\mathrm{keV} \gamma$ ray in our data since the former can only be produced from the $\beta$-decay of ${ }^{197} \mathrm{Pt}^{m}$ and the latter only from its $M 4$ transition. The efficiency-corrected ratio we obtain is $I_{\gamma 279} / I_{\gamma 346.5}=0.231(2)$. Using this result, together with the known relative intensities of other weaker $\gamma$ rays in ${ }^{197} \mathrm{Au}$ [14], we calculate that $1.9(1) \%$ of the total decay rate in the energy region of the $K$ x rays is due to the $\beta$ decay of ${ }^{197} \mathrm{Pt}^{m}$. This correction factor appears as well in Table II.

\section{Coincident summing}

It can be seen in Fig. 2 that the $346.5-\mathrm{keV}$ transition from ${ }^{197} \mathrm{Pt}^{m}$ is in cascade with a $53.1-\mathrm{keV}$ transition to the ground state of ${ }^{197} \mathrm{Pt}$. The latter is strongly converted $(\alpha=86.3)$ in the $L$ and higher shells, which leads in particular to platinum $L \mathrm{x}$ rays $(E=\sim 10 \mathrm{keV})$ being emitted in coincidence both with the $346.5-\mathrm{keV} \gamma$ rays and with the $K \mathrm{x}$ rays from the isomeric transition.

The coincident $\gamma-L$ sum peak is clearly visible to the right of the 346.5-keV peak in Fig. 3(b). We obtained the area of this sum peak in the first nine measured spectra and determined that its ratio to the $346.5-\mathrm{keV}$ peak area was independent of the decay rate for ${ }^{197} \mathrm{Pt}^{m}$ as expected for a coincident sum peak. The average value of the ratio for these nine spectra was then used to correct the areas for the $346.5-\mathrm{keV}$ peak, adding back the intensity lost to coincident summing. The correction factor we used is listed in Table II.

The same coincident summing must also occur with the $K \mathrm{x}$ rays from the isomeric transition but these sum peaks were already included in the total area that we obtained for the $K \mathrm{x}$-ray region of each spectrum. Therefore no correction for summing was required for the $\mathrm{x}$-ray decay rates.

Since the $K_{\alpha} \mathrm{x}$ ray from the isomeric transition can also occur in coincidence with an $L \mathrm{x}$ ray from the same transition, it is therefore possible that a triple sum will occur: one $K_{\alpha}$ with two $L$ x rays. This triple-sum peak would lie at the same energy as the double x-ray sum peak, $K_{\beta}+L$, and consequently would also lie within our defined $K$ x-ray region. Furthermore, our efficiency for detecting a $\sim 10-\mathrm{keV} L \mathrm{x}$ ray is $<1 \%$, so any triple-sum events would only appear at the $\ll 0.01 \%$ level, well below our measurement precision.

\section{E. Attenuation in the sample}

As described in Sec. III, our source was activated platinum grains in a layer that was (on average) $\sim 4.5 \mu \mathrm{m}$ thick, covered by a $12.5-\mu \mathrm{m}$-thick Mylar foil. For this configuration, we calculated the attenuation both of the platinum $\mathrm{x}$ rays and of the $346.5-\mathrm{keV} \gamma$ ray using standard tables of attenuation coefficients [19]. Since we are aiming at the evaluation of $\alpha_{K}$ from Eq. (1), what is important in that context is the attenuation for the $\mathrm{x}$ rays relative to that for the $\gamma$ ray. We calculated that the $\mathrm{x}$ rays suffered 1.4(5)\% more attenuation than the $\gamma$ ray and it is this result which is incorporated into Table II.

\section{F. Lorentzian correction}

As described in Sec. IV A, to be consistent with our previous efficiency-calibration procedures, we extracted our experimental peak areas using a special modification of the GF2 program that allows us to integrate the total counts above background within selected energy limits. To correct for possible missed counts outside those limits, the program adds an extrapolated Gaussian tail. We have noted in previous papers [5-8] that this extrapolated tail does not do full justice to $x$-ray peaks, whose shapes reflect the finite widths of the atomic levels responsible for them. To correct for this effect we computed simulated spectra using realistic Voigt-function shapes for the x-ray peaks and analyzed them with GF2 following exactly the same fitting procedure as was used for the real data to ascertain how much was missed by this approach.

One of the cases we studied previously was the decay of ${ }^{193} \mathrm{Ir}^{m}$ [5], in which we determined what correction needed to be applied to the area we obtained for iridium $\mathrm{x}$ rays in order to properly account for the Voigt shape. Since platinum and iridium are neighboring elements with $\mathrm{x}$ rays at very similar energies, we used the same analysis to establish that in the present case we lose $0.8(2) \%$ from the combined $\left(K_{\alpha}+K_{\beta}\right)$ $\mathrm{X}$-ray peaks of platinum. The corresponding correction factor appears in Table II.

\section{RESULTS AND DISCUSSION}

The various correction terms and the results of our analysis are given in Table II, where the top and middle sections of the table deal separately with the $K$ x rays and the $346.5-\mathrm{keV}$ $\gamma$ ray. With their decay rates both determined, we are now in a position to obtain the $\alpha_{K}$ value for the $346.5-\mathrm{keV} M 4$ transition by applying Eq. (1). The input data required to do so appear in the bottom section of the table together with our final result for the $K$-conversion coefficient itself, $\alpha_{K}=4.23(7)$.

In comparing our result to previous measurements, we refer to the Raman et al. survey [3] of world data on ICC values, from which we extracted the data presented in Fig. 1. The survey lists two previous measurements of $\alpha_{K}$ for the $346.5-\mathrm{keV}$ transition in ${ }^{197} \mathrm{Pt}$. The first, published in 1965 [20], gave the value $\alpha_{K}=3.9(4)$, which agrees with our result, albeit with a much larger uncertainty. The second measurement [15] was more recent by 20 years and its result, $\alpha_{K}=4.02(8)$, had a much smaller uncertainty, so it was the result that Raman et al. chose to adopt for their survey. We differ from this value by $5 \%$, or $3 \sigma$. 
TABLE III. Comparison of the measured $\alpha_{K}$ values for the $M 4$ transition from ${ }^{197} \mathrm{Pt}^{m}$ with calculated values based on different theoretical models for dealing with the $K$-shell vacancy. Shown also are the percentage deviations, $\Delta$, from the experimental value calculated as (experiment-theory)/theory. For a description of the various models used to determine the conversion coefficients, see Ref. [5].

\begin{tabular}{lcc}
\hline \hline Model & $\alpha_{K}$ & $\Delta(\%)$ \\
\hline Experiment & $4.23(7)$ & \\
Theory: & & \\
$\quad$ No hole & $4.191(10)$ & $+0.9(17)$ \\
Hole, frozen orbitals & $4.276(10)$ & $-1.1(17)$ \\
Hole, SCF of ion & $4.265(10)$ & $-0.8(17)$ \\
\hline \hline
\end{tabular}

To explain the discrepancy we need perhaps do no more than describe the contents of Ref. [15]. It is a three-page paper that reports ICC measurements of four $M 4$ transitions, all with quoted uncertainties less than $2 \%$. The single paragraph devoted to their ${ }^{197} \mathrm{Pt}^{m}$ measurement is seven lines long and makes no mention of the $\beta$-decay branch from the isomer nor any of the other corrections we deal with at length in Sec. IV. A glance at our Tables I and II shows that the neglect of some or all of the correction terms listed there could easily account for the earlier result being wrong by $5 \%$.

Our measured $\alpha_{K}$ value is compared with three different theoretical calculations in Table III. All three calculations are made within the Dirac-Fock framework, but one ignores the presence of the $K$-shell hole while the other two include it using different approximations: the frozen orbital approximation, in which it is assumed that the atomic orbitals have no time to rearrange after the electron's removal; and the SCF approximation, in which the final-state continuum wave function is calculated in the self-consistent field (SCF) of the ion, assuming full relaxation of the ion orbitals. To obtain these results we used the value $346.5(2) \mathrm{keV}$ [14] for the ${ }^{197} \mathrm{Pt}^{m}$ transition energy. The experimental uncertainty in this number is reflected in the uncertainties quoted on the theoretical values of $\alpha_{K}$ in the table.

The percentage deviations given in Table III indicate good agreement with all three calculations. The ${ }^{197} \mathrm{Pt}^{m}$ transition is not one characterized by a large difference between the $\alpha_{K}$ values calculated from the different theoretical approaches. Furthermore, the experimental complications of this measurement caused our experimental uncertainty to be a factor of two larger than we could achieve in our earlier measurements $[5,8]$. We cannot therefore claim a preference for any one of the three theoretical approaches.

Nevertheless our result clearly demonstrates that there is nothing anomalous about this $M 4$ transition. That point is convincingly illustrated by Fig. 6, in which our measurement (open circles labeled ${ }^{197} \mathrm{Pt}^{m}$ ) is compared graphically with two of the calculations and with the previously accepted experimental value (grey points). Where the ${ }^{197} \mathrm{Pt}^{m} \alpha_{K}$ value previously disagreed with all three Dirac-Fock calculations, our value is now entirely consistent with theory.

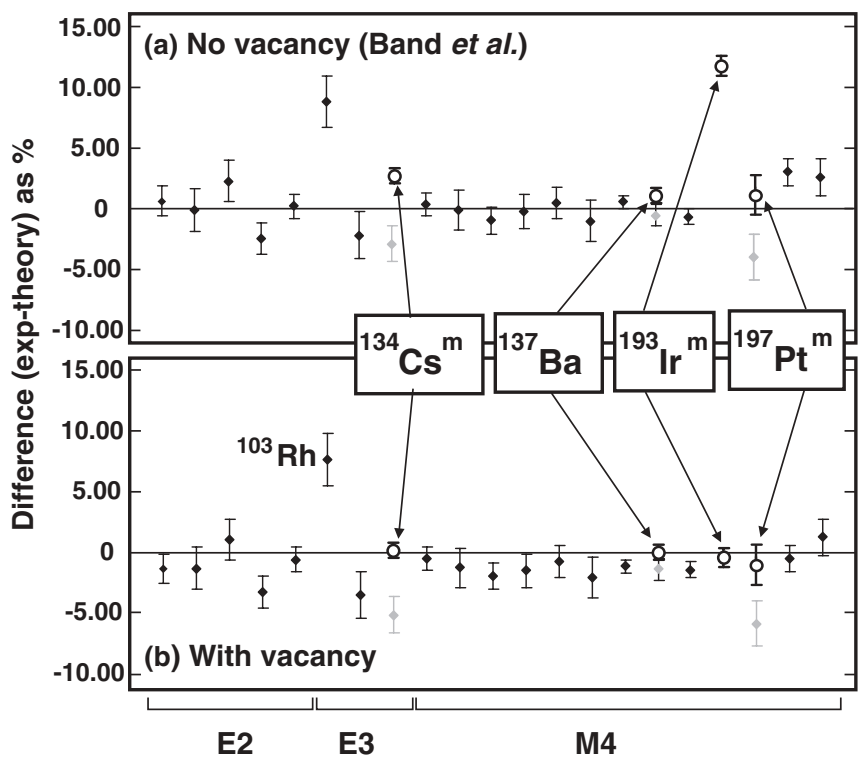

FIG. 6. The same plot as in Fig. 1 except that the results from the present work, for ${ }^{197} \mathrm{Pt}^{m}$, have been added as open circles. The previous values, which were listed by Raman et al. [3], are shown in grey.

\section{CONCLUSIONS}

Our measurement of the $K$-shell internal conversion coefficient, $\alpha_{K}$, for the $346.5-\mathrm{keV} M 4$ transition from ${ }^{197} \mathrm{Pt}^{m}$ has obtained a value that is significantly different from the only previous measurement quoted with comparable precision. Our new value is consistent with the Dirac-Fock theory and convincingly removes one of the few remaining discrepancies between an experimental ICC and theory.

A look at Fig. 6 will give the reader an appreciation of the current situation. Several years ago, our measurement of the $80.2-\mathrm{keV} M 4$ transition in ${ }^{193} \operatorname{Ir}[5,6]$ was the first to show definitively that the atomic vacancy must be included in the theory, at least in the case of low-energy $(\sim 4 \mathrm{keV})$ $K$-conversion electrons. Our later measurements of transitions in ${ }^{134} \mathrm{Cs}$ and ${ }^{137} \mathrm{Ba}[7,8]$ represented the first test among lighter nuclei and for transitions with significantly higher-energy conversion electrons. They confirmed our earlier conclusions by showing a clear preference for the Dirac-Fock theory that included provision for the atomic vacancy; and they also removed an apparent anomaly for the case of ${ }^{134} \mathrm{Cs}^{m}$ by replacing earlier faulty experimental results. Although our new result for ${ }^{197} \mathrm{Pt}^{m}$ does not help to distinguish among the theoretical approaches within the Dirac-Fock framework, it does remove an even larger experimental anomaly than the ${ }^{134} \mathrm{Cs}^{m}$ one. As a consequence, we can conclude with increased confidence that the Dirac-Fock calculations (including the atomic vacancy) provide a reliable way to determine ICCs.

\section{ACKNOWLEDGMENTS}

We thank Prof. M. P. Soriaga of the Chemistry Department at Texas A\&M University who suggested the chemical 
procedure we used to produce our platinum source. The work of the Texas A\&M authors is supported by the US Department of Energy under Grant No. DE-FG03-93ER40773 and by the
Robert A. Welch Foundation under Grant No. A-1397. The work of M.T. is supported by the Russian Foundation for Basic Research under Grant No. 05-02-17340.
[1] S. Raman, T. A. Walkiewicz, R. Gunnink, and B. Martin, Phys. Rev. C 7, 2531 (1973).

[2] R. S. Hager and E. C. Seltzer, Nucl. Data, Sect. A 4, 1 (1968).

[3] S. Raman, C. W. Nestor Jr., A. Ichihara, and M. B. Trzhaskovskaya, Phys. Rev. C 66, 044312 (2002); see also the electronic addendum to this paper, the location of which is given in the paper's Ref. [32].

[4] I. M. Band, M. B. Trzhaskovskaya, C. W. Nestor Jr., P. Tikkanen, and S. Raman, At. Data Nucl. Data Tables 81, 1 (2002).

[5] N. Nica, J. C. Hardy, V. E. Iacob, S. Raman, C. W. Nestor Jr., and M. B. Trzhaskovskaya, Phys. Rev. C 70, 054305 (2004).

[6] N. Nica, J. C. Hardy, V. E. Iacob, J. R. Montague, and M. B. Trzhaskovskaya, Phys. Rev. C 71, 054320 (2005).

[7] N. Nica, J. C. Hardy, V. E. Iacob, W. E. Rockwell, and M. B. Trzhaskovskaya, Phys. Rev. C 75, 024308 (2007).

[8] N. Nica, J. C. Hardy, V. E. Iacob, C. Balonek, and M. B. Trzhaskovskaya, Phys. Rev. C 77, 034306 (2008).

[9] J. H. Hubbell, P. N. Trehan, N. Singh, B. Chand, D. Mehta, M. L. Garg, R. R. Garg, S. Singh, and S. Puri, J. Phys. Chem. Ref. Data 23, 339 (1994).

[10] E. Schönfeld and H. Janssen, Nucl. Instrum. Methods Phys. Res. A 369, 527 (1996).
[11] J. C. Hardy, V. E. Iacob, M. Sanchez-Vega, R. T. Effinger, P. Lipnik, V. E. Mayes, D. K. Willis, and R. G. Helmer, Appl. Radiat. Isot. 56, 65 (2002).

[12] R. G. Helmer, J. C. Hardy, V. E. Iacob, M. Sanchez-Vega, R. G. Neilson, and J. Nelson, Nucl. Instrum. Methods Phys. Res. A 511, 360 (2003).

[13] R. G. Helmer, N. Nica, J. C. Hardy, and V. E. Iacob, Appl. Radiat. Isot. 60, 173 (2004).

[14] Huang Xiaolong and Zhou Chunmei, Nucl. Data Sheets 104, 283 (2005).

[15] I. N. Vishnevsky, V. A. Zheltonozhsky, and M. A. Ukhin, Izv. Acad. Nauk. SSSR, Ser. Fiz 51, 863 (1987) [Bull. Acad. Sci. USSR, Phys. Ser. 51, No 5, 23 (1987)].

[16] R. Jenkins, R. W. Gould, and D. Gedcke, Quantitative X-ray Spectroscopy (Marcel Dekker, New York, 1981), p. 266.

[17] D. Radford, http://radware.phy.ornl.gov/main.html; private communication.

[18] R. B. Firestone and L. P. Ekström, http://ie.lbl.gov/toi.

[19] C. T. Chantler, K. Olsen, R. A. Dragoset, J. Chang, A. R. Kishore, S. A. Kotochigova, and D. S. Zucker (2005), X-Ray Form Factor, Attenuation and Scattering Tables (version 2.1), available online at http://physics.nist.gov/ffast.

[20] A. J. Haverfield, H. T. Easterday, and J. M. Hollander, Nucl. Phys. 64, 379 (1965). 